\title{
Saint-Vivant de Vergy : un prieuré clunisien au cœur de la Bourgogne
}

A. RAUWEL (coord.), Saint-Vivant de Vergy : un prieuré clunisien au cœur de la Bourgogne, Curtil-Vergy, éd. association « L'abbaye de SaintVivant ", 2010, 203 p.

\section{Alain Rauwel}

\section{OpenEdition}

\section{Journals}

\section{Édition électronique}

URL : https://journals.openedition.org/cem/12075

DOI : $10.4000 /$ cem. 12075

ISSN : 1954-3093

Éditeur

Centre d'études médiévales Saint-Germain d'Auxerre

Édition imprimée

Pagination : 459-462

ISSN : $1623-5770$

Référence électronique

Alain Rauwel, «Saint-Vivant de Vergy : un prieuré clunisien au cœur de la Bourgogne », Bulletin du centre d'études médiévales d'Auxerre | BUCEMA [En ligne], 15 | 2011, mis en ligne le 25 août 2011, consulté le 22 septembre 2022. URL : http://journals.openedition.org/cem/12075; DOI : https:// doi.org/10.4000/cem.12075

Ce document a été généré automatiquement le 22 septembre 2022.

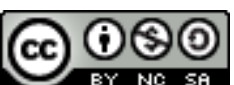

Creative Commons - Attribution - Pas d'Utilisation Commerciale - Partage dans les Mêmes Conditions 4.0 International - CC BY-NC-SA 4.0

https://creativecommons.org/licenses/by-nc-sa/4.0/ 


\section{Saint-Vivant de Vergy : un prieuré clunisien au cœur de la Bourgogne}

A. RAUWEL (coord.), Saint-Vivant de Vergy : un prieuré clunisien au cœur de la Bourgogne, Curtil-Vergy, éd. association « L'abbaye de SaintVivant ", 2010, 203 p.

\section{Alain Rauwel}

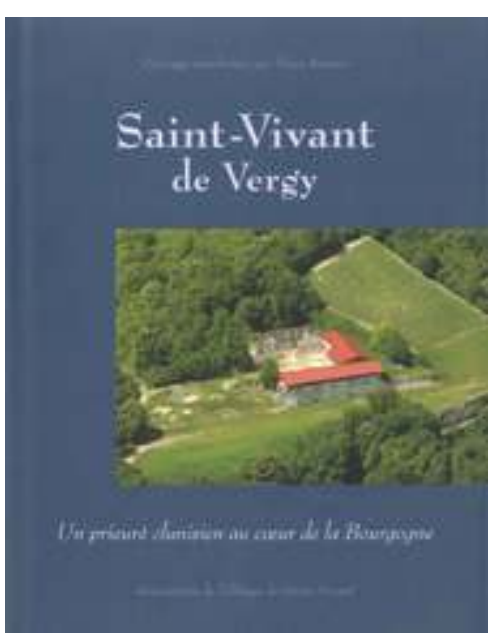

1 Curieusement, l'un des plus anciens et des plus riches monastères de Bourgogne, SaintVivant de Vergy, n'a, jusqu'à une date récente, guère attiré les historiens. La brochure exceptionnellement documentée et maîtrisée - du regretté chanoine Marilier demeurait depuis 1977 la référence obligée ${ }^{1}$. C'est le constat d'une certaine effervescence autour du prieuré de l'Arrière-Côte, qui a fait naître, un quart de siècle après, l'idée d'un ouvrage de synthèse. Le point de départ est à chercher dans le rachat des ruines de Saint-Vivant par une dynamique association, en 1996. À ce moment ont commencé des travaux de protection, de consolidation et de mise en valeur du site, qui sont aujourd'hui en voie d'achèvement. Les vestiges modernes ont ainsi, par la force des choses, été mieux étudiés et mieux compris. Les propriétaires et l'architecte en charge du chantier ont souhaité profiter de ces travaux pour lancer quelques sondages 
archéologiques, naturellement confiés au CEM : nouvelle occasion de progresser dans la connaissance des bâtiments ${ }^{2}$. Aux archives du sol, l'association «L'abbaye de SaintVivant" a tenu à joindre les archives de papier: mission a été confiée à Romuald Pinguet de dépouiller et d'inventorier le vaste fonds $21 \mathrm{H}$ des archives de la Côte-d'Or, superficiellement égratigné par les érudits antérieurs ${ }^{3}$; un répertoire a pu être établi. Par un très heureux hasard, cela a coïncidé avec la recherche doctorale de R. Pinguet, précisément centrée sur les domaines ecclésiastiques de cette région. Il y avait là un faisceau d'enquêtes convergentes, dont il eût été dommage de ne pas tirer parti.

2 Tout naturellement, l'histoire du prieuré commence par celle de son vocable. Bien que Vivant soit devenu l'un des prénoms les plus caractéristiques de la Bourgogne du Sud que l'on pense à Vivant Denon, ou au recueil de Roupnel Hé, Vivant !-, le saint patron est tout sauf indigène. La contribution introductive - A. Rauwel, « Reliques et légendes : les pérégrinations de saint Vivant» - rappelle les origines vendéennes du confesseur vénéré sur la butte de Vergy et suit le parcours, somme toute classique, des moines et des reliques au temps des invasions, d'Auvergne en Comté, puis en pays nuiton. Les acteurs des translations successives, par les parentés que l'on peut supposer, éclairent sur l'insertion de la communauté dans les réseaux aristocratiques pré-féodaux et féodaux. Les textes hagiographiques peuvent être, eux aussi, mis en relation avec les étapes du parcours.

3 Une fois les religieux installés sur leur éperon rocheux, il leur fallut construire un monastère - C. Sapin, «Les constructions monastiques médiévales de Saint-Vivant de Vergy ». L'archéologie nourrissait de grands espoirs, avant que les premiers coups de pelle des sondages soient donnés. Il a fallu en rabattre: les reconstructeurs du XVIII siècle n'ont pas fait les choses à moitié et les bâtiments médiévaux ont été rasés jusqu'à la roche avant le nouveau chantier. Même en négatif, on devine donc plus qu'on ne voit le Saint-Vivant clunisien. Heureusement, un plan très précis levé en 1762 permet de connaître les espaces monastiques anciens et de comparer Vergy à d'autres établissements de moines noirs.

4 «Saint-Vivant, monastère clunisien»: c'est la grande historienne des maisons clunisiennes, Denyse Riche, qui avait déjà évoqué le prieuré dans son maitre-livre ${ }^{4}$, qui a bien voulu affronter cette question centrale. A priori, le rattachement n'était pas des plus flatteurs, qui ramenait à l'état de dépendance une ancienne abbaye de plein exercice. Mais Vergy ne fut jamais, sur la carte des possessions de Cluny, un prieuré tout à fait comme les autres; sa singularité trouva à s'exprimer, fondée tant sur des origines vénérables que sur une indubitable prospérité foncière. Pain bénit pour l'historien, les comptes rendus de visite des définiteurs du chapitre général permettent de connaître, à partir du XIII siècle, l'état matériel et canonique de la communauté.

5 On a parlé de prospérité : l'abondance exceptionnelle des archives domaniales de SaintVivant a permis, pour la première fois, de préciser la nature et l'étendue des biens acquis et possédés - R. Pinguet, «Terres et territoires du prieuré de Saint-Vivant, $\mathrm{XII}^{\mathrm{e}}$ XVIII ${ }^{\mathrm{e}}$ siècle ». L'auteur était attendu au tournant par les historiens de la vigne et du vin, fascinés par le prestige de Vosne et de la Romanée-Saint-Vivant ${ }^{5}$; ils découvriront, de fait, les premières mentions de vignes, les dons, les aménagements, le développement de la micro-toponymie autour de ce qu'on appelle aujourd'hui, par pur effet de mode, les «climats». Mais il est permis de penser que l'étude des domaines forestiers de Saint-Vivant est plus novatrice encore: R. Pinguet examine ici l'étendue et, surtout, l'exploitation d'un bel ensemble de bois, qui fournissait aux moines une part 
importante de leurs revenus. Il apporte ainsi une contribution de première main à l'histoire économique de cette singulière région de l'Arrière-Côte, où la sylviculture et l'élevage ont joué un rôle au moins aussi important que la vigne. C'est au même jeune chercheur que l'on doit l'évocation de "La communauté sous l'Ancien Régime », qui donne un peu de chair à un récit davantage tourné, documentation oblige, vers les biens que vers les hommes.

6 Le paradoxe de Saint-Vivant est que sa gloire est médiévale, mais que l'on ne voit rien de médiéval sur le site. À partir de 1766, en effet, selon un processus très commun dans les monastères de l'Ancien Régime finissant - Cluny et Cîteaux, pour ne citer que ces exemples -, les religieux entreprennent, sous la direction de Caristie, une reconstruction intégrale de leur maison et de ses dépendances. L'histoire n'a pas permis que survivent les majestueux bâtiments élevés alors, mais les vestiges qui en subsistent et les deux étages de caves voûtées, encore en élévation, en donnent une grande idée. Il revenait naturellement à Christian Laporte, l'architecte qui veille depuis des années sur le chantier de Saint-Vivant, d'évoquer ces pierres qu'il connaît intimement ${ }^{6}$ (" $\mathrm{XvIII}^{\mathrm{e}}$ siècle : la grande reconstruction »). Il le fait en homme de l'art, dans un dialogue efficace avec les clichés pris par Henri Gaud spécialement pour cet ouvrage.

7 Peut-être les usages académiques eussent-ils conduit à s'en tenir là ${ }^{7}$. Mais, comme Benoît Chauvin l'a fait récemment pour le Clos-Vougeot ${ }^{8}$, les auteurs ont tenu à poursuivre jusqu'à aujourd'hui l'histoire du site. Jean-François Bazin, dont l'intérêt pour Vergy ne date pas d'hier, a accepté de retracer «Le destin d'une ruine romantique ». Destin mouvementé, en vérité, éclairé par une restitution rigoureuse de la succession des propriétaires et par le recours à une belle série de documents iconographiques inédits, qui permettent de suivre la lente dégradation des bâtiments et leur envahissement par la végétation - et aussi, par contraste, l'efficacité des travaux récents, qui n'autorisent peut-être plus à parler, comme le fait joliment J.-F. Bazin pour la Belle Époque, de "promenade au pays perdu ", mais qui ont rendu tout le lustre possible à un point majeur de la carte monastique de la Bourgogne.

Est-ce à dire que le volume offert au public en décembre 2010 représente une somme définitive sur Saint-Vivant? Nul ne saurait le prétendre. Six historiens y font le point sur l'état actuel des connaissances, et ce bilan méritait d'être proposé, car beaucoup d'avancées ont eu lieu depuis l'esquisse pionnière de Jean Marilier. Mais le travail continue, et il faudra sans doute, un jour, un autre Saint-Vivant. De nouveaux sondages archéologiques vont avoir lieu dans des zones moins touchées par l'arasement moderne. Certains secteurs, seulement indiqués en 2010, comme la remarquable bibliothèque du prieuré, profitent déjà de découvertes nouvelles ${ }^{9}$, destinées à se multiplier. Et Saint-Vivant ne pourra que tirer parti de recherches d'ensemble sur la colline de Vergy, qui amèneront à mettre le monastère en situation, dans les échanges serrés qu'il entretenait avec le château, le bourg et sa rivale ecclésiastique, la collégiale Saint-Denis ${ }^{10}$.

9 1. . J. MARILIER, Le Monastère de Saint-Vivant de Vergy, 1977 (Cahiers de Vergy, 1), rééd. en 1999.

10 2. . Cf. Bucema, 10 (2006), p. 63-64 [http://cem.revues.org/index154.html].

11 3. À l'exception de Jules d'Arbaumont, qui avait préparé un cartulaire de Saint-Vivant demeuré manuscrit (ADCO, $12 \mathrm{~J})$, et de Maurice Chaume, qui avait transcrit des actes du 
prieuré en vue de ce grand cartulaire bourguignon, qu'une mort prématurée l'a empêché de donner et dont le regret point tous les diplomatistes.

4. . L'Ordre de Cluny à la fin du Moyen Âge : le vieux pays clunisien, XII ${ }^{e}-X V^{e}$ siècle, SaintÉtienne, 2000.

5. . Ce clos légendaire fait dans l'ouvrage l'objet d'un chapitre additionnel, dû à la plume experte de J.-F. Bazin. Cf. aussi R. PINGUET, «Les vignobles du grand prieuré clunisien de Saint-Vivant de Vergy ", in La vigne et les hommes en Bourgogne et alentour, l'histoire de la mise en valeur des territoires, Beaune, 2007, p. 29-46.

6. . Cf. déjà C. LAPORTE et R. GRIMA, « Les bâtiments du XVIII siècle à Saint-Vivant de Vergy et leur restauration ", Mémoires de la Commission des antiquités de la Côte-d'Or, 40 (2002-2004), p. 233-250.

15 7. . Sur la période révolutionnaire, cf. R. PINGUET, «Les derniers religieux de SaintVivant", in Citoyennes et citoyens de Côte-d'Or en Révolution, 2010 (Cahiers du Comité départemental pour l'histoire de la Révolution en Côte-d'Or, 3), p. 173-181.

8. . B. CHAUVIN, Le Clos et le château de Vougeot, cellier de l'abbaye de Cîteaux, Nuits-SaintGeorges, 2008.

9. . M. DESACHY, «Bibliophiles d'oncle à neveu: livres et bibliothèques de Jean et Hélion Jouffroy (vers 1460-1530) », Bulletin du bibliophile, 2010, p. 36-60.

18 10. . V. TABBAGH, «Construction sacrée, réforme spirituelle et vénération des saints au milieu du XV $\mathrm{XV}^{\mathrm{e}}$ siècle : l'exemple de la collégiale de Vergy ", in Les clercs, les fidèles et les saints en Bourgogne médiévale, Dijon, 2005, p. 115-130.

\section{INDEX}

Mots-clés : prieuré

Index géographique : France/Saint-Vivant de Vergy 